\title{
Online Appendix for Risk Premia and the Real Effects of Money
}

\author{
By Sebastian Di Tella
}

This Online Appendix contains omitted proofs, the extension with EZ preferences, and the contractual environment.

\section{Ommited Proofs}

\section{PROOF OF PROPOSITION 1:}

The properties of $\lambda$ and $\sigma_{c}$ are straightforward from equation (8), noticing only that $\rho-((1-\lambda) \sigma)^{2}=r-(\hat{x}-\delta)>0$ in equilibrium. First, for $\beta>0, \lambda \in(0,1)$ because $k_{t}$, and $m_{t}+h_{t}$ are strictly positive. The left hand side of (8) is increasing in $\lambda$ while the right hand side is decreasing. Since the right hand side is increasing in $\sigma$, it follows that $\lambda$ is increasing in $\sigma$. When $\sigma=0$, (8) simplifies to $\lambda=\beta$, while in the limit as $\sigma \rightarrow \infty$, since $\lambda>0$ we must have $(1-\lambda) \rightarrow 0$.

The right hand side is increasing in the idiosyncratic consumption risk $\sigma_{c}=$ $(1-\lambda) \sigma$, so since $\lambda$ is increasing in $\sigma$, so must $\sigma_{c}$. When $\sigma=0$ we have $\lambda=\beta$, so $\sigma_{c}=0$. When $\sigma \rightarrow \infty$ we have $\lambda \rightarrow 1$, so $\sigma_{c}=(1-\lambda) \sigma \rightarrow \sqrt{\rho(1-\beta)}>0$.

For $\beta=0$ we clearly have $\lambda=0$ from (11).

\section{PROOF OF PROPOSITION 2:}

Straightforward from equilibrium conditions $r=a-\delta-\sigma^{2}$ and $\hat{x}=a-\rho$.

\section{PROOF OF PROPOSITION 3:}

We know from Proposition 1 that $\lambda$ and $\sigma_{c}=(1-\lambda) \sigma$ are increasing in $\sigma$. So the real interest rate $r=a-\delta-(1-\lambda) \sigma^{2}$ falls when $\sigma$ increases, but less so than without money, since in that case $r=a-\delta-\sigma^{2}$. Investment $\hat{x}$ falls because the term $\rho \frac{\lambda-\beta}{1-\lambda}>0$ is increasing in $\lambda$.

If $\sigma=0$, we have $r=a-\delta, \lambda=\frac{\rho \beta}{\rho}=\beta$, and $\hat{x}=a-\rho$ which coincide with the non-monetary economy.

\section{PROOF OF PROPOSITION 4:}

The inflation target $\pi$ does not appear in equations (14), (15), and (8) for $r, \hat{x}$, and $\lambda$. It only appears in equation (9) for $\hat{m}$.

Targeting the nominal interest rate is accomplished by picking the inflation target $\pi$ so that $i=r+\pi$ is constant. Since $\pi$ does not affect $r$, or any other real variable, we can do this to hit any $i>0$. Notice that targeting $i$ introduces the usual indeterminacy in the price level, since only the expected inflation is pinned down. 
Finally, since nothing real is affected by changing the inflation target, and $i \approx 0$ maximizes the utility from money services $m$, the optimal inflation target delivers the Friedman rule.

\section{PROOF OF PROPOSITION 5:}

From individual optimization we know that the expenditure share of liquidity is $\beta$, invariant to $b$. Since $r, \hat{x}$, and $\lambda$ are pinned down by (14), (15), and (8), they are not affected by changes in $b$.

\section{PROOF OF PROPOSITION 6:}

It suffices to look at the behavior of $\lambda$ defined by (8), which we can rewrite

$$
\lambda\left(\rho-((1-\lambda) \sigma)^{2}\right)=\rho \beta
$$

As $\beta \rightarrow 0$ the left hand side converges to zero, and so must the right hand side. This means that either a) $\lambda \rightarrow 0$ or b) $\lambda \rightarrow 1-\frac{\sqrt{\rho}}{\sigma}$. If $\sigma<\sqrt{\rho}$, then $1-\frac{\sqrt{\rho}}{\sigma}<0$. Since $\lambda>\beta$ always, b) cannot be, so we are left with a) $\lambda \rightarrow 0$. From (14) and (15) we see that $r$ and $x$ converge to the values on the non-monetary economy with $\lambda=\beta=0$.

If instead $\sigma \geq \sqrt{\rho}$, we cannot have $\lambda \rightarrow 0$, because it implies that $\rho-((1-$ $\lambda) \sigma)^{2} \leq 0$ at some point along the way (for $\lambda$ small enough). Since $\lambda>\beta$ always, this requires $\rho \beta<0$, which is not true. So we have b) $\lambda \rightarrow 1-\frac{\sqrt{\rho}}{\sigma} \geq 0$, and the inequality is strict if $\sigma>\sqrt{\rho}$. From (14) and (15) we see that the real interest rate $r$ is high and investment $\hat{x}$ low relative to the economy without money $(\beta=0)$.

\section{PROOF OF PROPOSITION 7:}

See Theorem 1 in Section III of this Online Appendix.

\section{PROOF OF PROPOSITION 8:}

Combine (28) with $\sigma_{c}=\frac{\rho(1-\beta) \sigma}{a-\hat{x}}$ to obtain

$$
\sigma_{c}=\frac{\rho(1-\beta)}{\rho+\sigma_{c}^{2}} \sigma
$$

It follows that when $\sigma=0$ we get $\sigma_{c}=0$, and $\sigma_{c}$ is increasing in $\sigma$. Rewrite it

$$
\frac{\sigma_{c}}{\sigma}=\frac{\rho(1-\beta)}{\rho+\left(\frac{\sigma_{c}}{\sigma}\right)^{2} \sigma^{2}}
$$

It follows that $\sigma_{c} / \sigma$ is decreasing in $\sigma$. The properties of $\hat{x}$ follow from equation (28). Finally, write

$$
r=\rho+\hat{x}-\delta-\sigma_{c}^{2}=a-\delta-2 \sigma_{c}^{2}
$$


It follows that $r$ falls with $\sigma$.

PROOF OF PROPOSITION 9:

First take the $\beta \in(0,1 / 2)$ case. Rewrite (29) and (30) in terms of $y=\sigma_{c} / \sigma$

$$
\begin{array}{lr}
\hat{x}_{S P}=a-\rho-y^{2} \sigma^{2} & \text { Social Planner } \\
\hat{x}_{C E}=a-\rho-y \sigma^{2} \times(1-y) & \text { Competitive Equilibrium }
\end{array}
$$

and the incentive-compatible combinations

$$
\hat{x}_{I C}=a-\frac{\rho(1-\beta)}{y}
$$

The competitive equilibrium lies at the intersection of $\hat{x}_{C E}$ and $\hat{x}_{I C}$; call the corresponding $y_{C E} \in[0,1-\beta]$. The planner's allocation lies at the intersection of $\hat{x}_{S P}$ and $\hat{x}_{I C}$, call the corresponding $y_{S P} \in[0,1-\beta]$.

We know that $y=\sigma_{c} / \sigma$ can range from 0 to $1-\beta$, in both the CE and SP (the upper bound comes from knowing that investment is below the first best in both the $\mathrm{CE}$ and the SP, and using $\hat{x}_{I C}$.

$\hat{x}_{I C}$ is increasing, strictly concave, and ranges from $-\infty$ when $y=0$ to the first best $a-\rho$ when $y=1-\beta$. It does not depend on $\sigma$, so it will be fixed when we do comparative statics.

$\hat{x}_{C E}$ and $\hat{x}_{S P}$ do depend on $\sigma$. They both start at the first best $a-\rho$ when $y=0 . \hat{x}_{S P}$ is strictly decreasing and concave (it's an inverted parabola) with vertex at $(0, a-\rho)$. So it must cross $\hat{x}_{I C}$ exactly once.

$\hat{x}_{C E}$ is a parabola with vertex at $\left(\frac{1}{2}, a-\rho-\frac{1}{4} \sigma^{2}\right)$. Importantly, it intersects with $\hat{x}_{S P}$ at this point. For $\sigma>0$ they intersect at exactly two points, corresponding to $y=0$ and $y=1 / 2$, and this implies that $\hat{x}_{C E}<\hat{x}_{S P}$ for all $y \in(0,1 / 2)$, and $\hat{x}_{C E}>\hat{x}_{S P}$ for all $y \in(1 / 2,1-\beta)$. Finally, $\hat{x}_{C E}<a-\rho$ for all $y \in(0,1-\beta)$. In particular, $\hat{x}_{C E}(1-\beta)=a-\rho-\sigma^{2} \beta(1-\beta)<a-\rho$. Since $\hat{x}_{C E}$ is strictly convex and $\hat{x}_{I C}$ is strictly concave they intersect at two points at most. Since $\hat{x}_{I C}=\infty$ for $y=0, \hat{x}_{I C}$ crosses $\hat{x}_{C E}$ first from below, and the from above. But since $\hat{x}_{I C}(1-\beta)=1-\rho>\hat{x}_{C E}(1-\beta)$, the second intersection has $y>1-\beta$, so it is not in the range on $y$. There is then only one valid intersection between $\hat{x}_{C E}$ and $\hat{x}_{I C}$; we called it $y_{C E} \in[0,1-\beta]$ and $\hat{x}_{I C}<\hat{x}_{C E}$ for all $y<y_{C E}$ and $\hat{x}_{I C}>\hat{x}_{S P}$ for all $y>y_{C E}$ in the range of $y$.

Now the lower envelope of $\hat{x}_{C E}$ and $\hat{x}_{S P}, \hat{x}_{L}=\min \left\{\hat{x}_{C E}, \hat{x}_{S P}\right\}$ coincides with $\hat{x}_{C E}$ for $y \in[0,1 / 2]$ and with $\hat{x}_{S P}$ for $y \in[1 / 2,1-\beta]$. This implies that if $\hat{x}_{I C}$ first intersects with the lower envelope for $y<1 / 2$, it must do so at $y_{C E}$, and if it first intersects at $y>1 / 2$, it must do so at $y_{S P}$. In the first case, since $\hat{x}_{S P}>\hat{x}_{C E}$ for $y<1 / 2$, it is strictly decreasing, and goes from $a-\rho$ for $y=0$ to $a-\rho-\frac{1}{4} \sigma^{2}=\min \hat{x}_{C E}$ for $y=1 / 2$; and $\hat{x}_{I C}$ is strictly increasing and goes to $1-\rho$; then it means that $y_{S P}<1 / 2$ as well and $y_{S P}>y_{C E}$. In the second case, obviously $y_{C E}>y_{S P}>1 / 2$. If it first intersects at $y=1 / 2$ then $y_{C E}=y_{S P}$. 
It follows immediately that if $y_{C E}<y_{S P}$ and both below $1 / 2$, then $\hat{x}_{C E}<\hat{x}_{S P}$ and $\sigma_{c}^{C E}<\sigma_{c}^{S P}$. On the other hand, if $y_{C E}>y_{S P}$ and both above $1 / 2$, then $\hat{x}_{C E}>\hat{x}_{S P}$ and $\sigma_{c}^{C E}>\sigma_{c}^{S P}$.

It only remains to see which will hold for a given $\sigma$. Since both $\hat{x}_{C E}$ and $\hat{x}_{S P}$ are decreasing for $y \in(0,1 / 2)$, and $\hat{x}_{I C}$ is always increasing, it is enough to compare their values at $y=1 / 2$. If $\hat{x}_{C E}=\hat{x}_{S P} \geq \hat{x}_{I C}$ at $y=1 / 2$, then $y_{C E}$ and $y_{S P}$ are both in $[1 / 2,1-\beta]$. If instead $\hat{x}_{C E}=\hat{x}_{S P} \leq \hat{x}_{I C}$ at $y=1 / 2$, then $y_{C E}$ and $y_{S P}$ are both in $(0,1 / 2)$.

$$
\begin{gathered}
\hat{x}_{C E}=\hat{x}_{S P} \geq \hat{x}_{I C} \\
\Longleftrightarrow a-\rho-\frac{1}{4} \sigma^{2} \geq a-2 \rho(1-\beta) \\
\Longleftrightarrow \sigma^{2} \leq \sigma^{*}=2 \sqrt{\rho(1-2 \beta)}>0
\end{gathered}
$$

Finally, for the case $\beta \in[1 / 2,1), \hat{x}_{C E}<\hat{x}_{S P}$ for all $y \in[0,1-\beta]$, regardless of $\sigma$, so $\hat{x}_{C E}<\hat{x}_{S P}$ and $\sigma_{c}^{C E}<\sigma_{c}^{S P}$. Notice that in this case the formula for $\sigma^{*}<0$.

\section{PROOF OF PROPOSITION 10:}

We already know that the planner's allocation is a BGP with constant $\hat{x}$ and $\sigma_{c}$. By setting the subsidy/tax $\tau^{k}$ according to (36) we ensure that $r, \hat{x}$, and $\hat{m}$ satisfy all the conditions for a BGP equilibrium.

We can check that the value of total wealth $w=k_{t}+m_{t}+h_{t}-\int_{t}^{\infty} e^{-r s} \tau_{s}^{k} k_{s} d s$ satisfies $c_{t}=\rho(1-\beta) w_{t}$, or equivalently $\sigma_{c}=\frac{k_{t}}{w_{t}} \sigma$. Write

$$
\begin{gathered}
\frac{w_{t}}{k_{t}}=1+\frac{\hat{m} i}{r-(\hat{x}-\delta)}+\frac{\tau^{k}}{r-(\hat{x}-\delta)} \\
\frac{w_{t}}{k_{t}}=\frac{r-(\hat{x}-\delta)+\hat{c} \frac{\beta}{1-\beta}+\tau^{k}}{r-(\hat{x}-\delta)}=\frac{\rho-\sigma_{c}^{2}+\rho \beta \frac{\sigma}{\sigma_{c}}+2 \sigma_{c}^{2}-\sigma_{c} \sigma}{\rho-\sigma_{c}^{2}} \\
\frac{w_{t}}{k_{t}}=\frac{\rho+\rho \beta \frac{\sigma}{\sigma_{c}}+\sigma_{c}^{2}-\sigma_{c} \sigma}{\rho-\sigma_{c}^{2}}=\frac{\sigma}{\sigma_{c}}\left(\frac{\rho \frac{\sigma_{c}}{\sigma}+\rho \beta+\sigma_{c}^{2}\left(\frac{\sigma_{c}}{\sigma}-1\right)}{\rho-\sigma_{c}^{2}}\right)
\end{gathered}
$$

Use the planner's FOC (29) and the skin in the game IC constraint (31)

$$
\begin{gathered}
\frac{w_{t}}{k_{t}}=\frac{\sigma}{\sigma_{c}}\left(\frac{\rho \frac{\sigma_{c}}{\sigma}+\rho \beta+(a-\hat{x}-\rho)\left(\frac{\sigma_{c}}{\sigma}-1\right)}{2 \rho-a-\hat{x}}\right)=\frac{\sigma}{\sigma_{c}}\left(\frac{\rho(1+\beta)+(a-\hat{x}) \frac{\rho(1-\beta)-(a-\hat{x})}{a-\hat{x}}}{2 \rho-a-\hat{x}}\right) \\
\frac{w_{t}}{k_{t}}=\frac{\sigma}{\sigma_{c}}\left(\frac{\rho(1+\beta)+\rho(1-\beta)-(a-\hat{x})}{2 \rho-a-\hat{x}}\right)=\frac{\sigma}{\sigma_{c}} \frac{2 \rho-(a-\hat{x})}{2 \rho-(a-\hat{x})}=\frac{\sigma}{\sigma_{c}}
\end{gathered}
$$

The optimal inflation target implements the Friedman rule and delivers unbounded utility from real money balances (in a supremum sense). 


\section{PROOF OF PROPOSITION 11:}

First, to implement the optimal allocation we need $r>\hat{x}-\delta$ which is equivalent to $\rho-\sigma_{c}^{2}>0$. We know $\sigma_{c}$ in the optimal allocation is given by

$$
\sigma_{c}=\frac{\rho(1-\beta)}{\rho+\sigma_{c}^{2}} \sigma
$$

and $\sigma_{c}$ is increasing in $\sigma$. For $\sigma=0$ we have $\sigma_{c}=0$ too, so $\rho-\sigma_{c}^{2}>0$. So we only need to ask at what $\sigma$ we have $\sigma_{c}^{2}=\rho$ :

$$
\sqrt{\rho}=\frac{\rho(1-\beta)}{\rho+\rho} \bar{\sigma} \Longrightarrow \bar{\sigma}=\frac{2 \sqrt{\rho}}{1-\beta}
$$

For $\sigma \geq \bar{\sigma}$ we have $\sigma_{c}^{2} \geq \rho$ and therefore $r \leq \hat{x}-\delta$, so the optimal allocation cannot be implemented as a competitive equilibrium with a tax on capital.

For the sign of $\tau^{k}$, use (36) to get

$$
\tau^{k}=\sigma_{c}\left(2 \sigma_{c}-\sigma\right)
$$

So if $\sigma_{c}>\frac{1}{2} \sigma$ we have $\tau^{k}>0$, and if $\sigma_{c}<\frac{1}{2} \sigma$ we have $\tau^{k}<0$. In the optimal allocation we have

$$
\frac{\sigma_{c}}{\sigma}=\frac{\rho(1-\beta)}{\rho+\left(\frac{\sigma_{c}}{\sigma}\right)^{2} \sigma^{2}}
$$

So $\sigma_{c} / \sigma \rightarrow 1-\beta$ when $\sigma \rightarrow 0$, and $\sigma_{c} / \sigma$ is decreasing in $\sigma$. So if $\beta \geq \frac{1}{2}$ we must have $\sigma_{c} \leq \frac{1}{2} \sigma$ and therefore $\tau^{k} \leq 0$, for all $\sigma \in[0, \bar{\sigma})$. If instead $\beta<\frac{1}{2}$, we have $\tau^{k}>0$ for $\sigma$ close to 0 . We only need to find $\sigma^{*}$ such that $\sigma_{c}=\frac{1}{2} \sigma^{*}$.

$$
\frac{1}{2}=\frac{\rho(1-\beta)}{\rho+\frac{1}{4}\left(\sigma^{*}\right)^{2}} \Longrightarrow \sigma^{*}=2 \sqrt{\rho(1-2 \beta)}
$$

It only remains to show that $\sigma^{*} \in(0, \bar{\sigma}] . \sigma^{*}>0$ follows from $\beta<\frac{1}{2}$. Now write

$$
\sigma^{*}=2 \sqrt{\rho(1-2 \beta)} \leq 2 \sqrt{\rho} \leq \frac{2 \sqrt{\rho}}{1-\beta}=\bar{\sigma}
$$

\section{PROOF OF PROPOSITION 12:}

From the definition of $h$,

$$
m_{t}+h_{t}=m_{t}+\mathbb{E}_{t}^{Q}\left[\int_{t}^{T} e^{-\int_{t}^{s} r_{u} d u} \frac{d M_{s}}{p_{s}}\right]+\mathbb{E}_{t}^{Q}\left[e^{-\int_{t}^{T} r_{u} d u} h_{T}\right]
$$


use $d m_{t}=\frac{d M_{t}}{p_{t}}-m_{t} \pi_{t} d t$ to write

$$
=m_{t}+\mathbb{E}_{t}^{Q}\left[\int_{t}^{T} e^{-\int_{t}^{s} r_{u} d u}\left(d m_{s}+\pi_{s} m_{s} d s\right)\right]+\mathbb{E}_{t}^{Q}\left[e^{-\int_{t}^{T} r_{u} d u} h_{T}\right]
$$

We can write

$$
e^{-\int_{t}^{T} r_{u} d u} m_{T}=m_{t}+\int_{t}^{T} e^{-\int_{t}^{s} r_{u} d u}\left(-r_{s} m_{s} d s+d m_{s}\right)
$$

and plug it in to obtain

$$
m_{t}+h_{t}=\mathbb{E}_{t}^{Q}\left[\int_{t}^{T} e^{-\int_{t}^{s} r_{u} d u}\left(\left(r_{s}+\pi_{s}\right) m_{s} d s\right)\right]+\mathbb{E}_{t}^{Q}\left[e^{-\int_{t}^{T} r_{u} d u}\left(m_{T}+h_{T}\right)\right]
$$

Then take the limit $T \rightarrow \infty$ and use the transversality condition

$$
\lim _{T \rightarrow \infty} \mathbb{E}_{t}^{Q}\left[e^{-\int_{t}^{T} r_{u} d u}\left(m_{T}+h_{T}\right)\right]=0
$$

and the monotone convergence theorem $\left(r_{t}+\pi_{t}=i_{t} \geq 0\right)$ to obtain

$$
m_{t}+h_{t}=\mathbb{E}_{t}^{Q}\left[\int_{t}^{\infty} e^{-\int_{t}^{s} r_{u} d u} m_{s} i_{s} d s\right]
$$

\section{PROOF OF PROPOSITION 13:}

From (49), we plug in $\theta^{T F P}=\tilde{\sigma}^{T F P}$, and $\theta^{R S}=\frac{\sigma_{\psi}}{1+\psi}$, as well as $\hat{m} i=\rho \beta w / k=$ $\rho \beta(1+\psi)$ from $(47)$ and

$$
\hat{x}+g-\delta-r=-\left(\rho+\frac{\mu_{\psi}}{1+\psi}-\left(\frac{\sigma}{1+\psi}\right)^{2}-\left(\tilde{\sigma}^{T F P}\right)^{2}-\left(\frac{\sigma_{\psi}}{1+\psi}\right)^{2}\right)
$$

Then use Ito's lemma to obtain

$$
\begin{gathered}
\mu_{\psi}=\psi^{\prime} \phi(\bar{\sigma}-\sigma)+\frac{1}{2} \psi^{\prime \prime} \sigma \nu^{2} \\
\sigma_{\psi}=\psi^{\prime} \sqrt{\sigma} \nu
\end{gathered}
$$

The ODE (51) has the $\mu_{\psi}$ terms together, and the $\tilde{\sigma}^{T F P}$ terms cancel out. I also simplified the terms involving $\sigma_{\psi}$ into one term.

\section{PROOF OF PROPOSITION 14:}

The equations for the competitive equilibrium are a modified version of (42)(47), taking into account that total wealth now includes the present value of 
taxes/subsidies that are rebated lump-sum and the tax $\tau^{k}$ :

$$
r_{t}=\rho+\left(\hat{x}_{t}+g-\delta\right)+\mu_{\hat{c}, t}-\sigma_{c t}^{2}-\left(\tilde{\sigma}_{c t}^{T F P}\right)^{2}-\left(\tilde{\sigma}_{c t}^{R S}\right)^{2}
$$

Euler

$$
\sigma_{c t}=\frac{k_{t}}{w_{t}} \sigma_{t}=(1-\beta) \rho k_{t} c_{t}^{-1} \sigma_{t}
$$

idiosyncratic risk

$$
\tilde{\sigma}_{c}^{T F P}=\theta_{t}^{T F P}
$$

TFP risk

$$
\tilde{\sigma}_{c t}^{R S}=\theta_{t}^{R S}
$$

$$
\hat{m}_{t}=\beta /(1-\beta) \times\left(a-\hat{x}_{t}\right) /\left(r_{t}+\pi_{t}\right)
$$

risk shock risk

Since the planner's allocation satisfies (52)-(57) and the FOC (59), it satisfies also the equilibrium conditions (A.1)-(A.6). Equations (A.1), (A.4), (A.5), and (A.6) are immediate. Equation (A.2) follows from plugging the definition of $\hat{x}_{t}$ from (59), $\tau_{t}^{k}$ from (60), and $\theta_{t}^{T F P}=\tilde{\sigma}_{c t}^{T F P}$ into the Euler equation (53). (A.3) comes from the skin in the game constraint (52), using the fact that $\sigma_{U t}=\frac{1}{\rho} \sigma_{c t}$. Finally, $\lim _{t \rightarrow \infty} \mathbb{E}^{Q}\left[e^{-\int_{0}^{t} r_{u} d u} k_{t} \hat{c}_{t}\right] \Longrightarrow \lim _{t \rightarrow \infty} \mathbb{E}^{Q}\left[e^{-\int_{0}^{t} r_{u} d u} w_{t}\right]=0$ and $i_{t}=r_{t}+\pi_{t}>0$ ensure that this is in fact an equilibrium.

\section{PROOF OF PROPOSITION 15:}

Let $g(n ; r)$ be the stationary distribution of wealth in the steady state. Market clearing in the steady state requires

$$
\int n g(n ; r) d n=k_{s s}(r)+m_{s s}(r)
$$

where $g, k_{s s}$, and $m_{s s}$ depend on the steady state real interest rate $\left(m_{s s}\right.$ depends on the nominal interest rate, but with constant money supply, inflation in the steady state is zero, so $i=r$ ). We must find $r$ that solves this equation. In general this requires finding the stationary distribution of wealth generated by each $r$.

Optimize $(1-\beta) \log c+\beta \log m$ subject to total expenditures $x=c+m i$ to obtain an indirect utility function. We obtain the usual demand for money

$$
m=\frac{\beta}{1-\beta} \frac{c}{i}
$$

and an indirect utility function $\tilde{u}(x)=\log x+A(i)$, for some constant function 
$A(i)$. We can then re-write the budget constraint

$$
d n_{i t}=\left(n_{i t} r+w(r) e_{i t}-x_{t}\right) d t
$$

We conclude that for a given $r$, we get the same distribution of wealth $g_{0}(n ; r)$ as in a model without money and log preferences only over consumption, $\beta=0$.

Market clearing with money requires

$$
\int n g_{0}(n ; r) d n-k_{s s}(r)=m_{s s}(r)
$$

Normalizing by the steady state wage $w_{s s}(r)=f_{l}^{\prime}\left(k_{s s}(r)\right)$, we get

$$
\frac{\int n g_{0}(n ; r) d n}{w_{s s}(r)}-\frac{k_{s s}(r)}{w_{s s}(r)}=\frac{m_{s s}(r)}{w_{s s}(r)}
$$

We can now compare the economy without money, $\beta=0$, to the economy with money, $\beta>0$. The distribution of wealth and the capital stock is the same in both cases, but with money we get a positive term on the left hand side. It's a standard result that with these preferences and technology, the right hand side is an increasing function of $r,{ }^{1}$ so we can conclude that in the monetary economy, the interest rate is higher, and the capital stock lower, relative to the non-monetary economy.

\section{The role of intertemporal elasticity and risk aversion}

The baseline model has log preferences, which yield clean results and are quantitatively reasonable. In this Appendix I extend the baseline model to allow for EZ preferences to understand the role of intertemporal elasticity and risk aversion.

Suppose agents have recursive EZ preferences with discount $\rho$, risk aversion $\gamma$, and intertemporal elasticity $\psi$. If $\psi=1 / \gamma$ we have the standard CRRA preferences. If $\psi=\gamma=1$ we have the baseline model with log preferences.

The equilibrium equations are now modified as follows

$$
\begin{array}{rlr}
r & =\rho+(\hat{x}-\delta) / \psi-(1+1 / \psi)(\gamma / 2) \sigma_{c}^{2} & \text { Euler equation } \\
r & =a-\delta-\gamma \sigma_{c} \sigma & \text { asset pricing } \\
\sigma_{c} & =(1-\lambda) \sigma & \text { risk sharing } \\
\hat{m} & =\frac{\beta}{1-\beta} \frac{a-\hat{x}}{r+\pi} & \text { money }
\end{array}
$$

The expression for the liquidity share, $\lambda$, must be solved simultaneously with $r$

${ }^{1}$ See for example Light (2018). 
and $\hat{x}$.

$$
\lambda=\frac{\rho \beta}{\rho+(1 / \psi-1)(\hat{x}-\delta)-(1+1 / \psi)(\gamma / 2)((1-\lambda) \sigma)^{2}}
$$

We can check that if $\psi=\gamma=1$ we recover the equation in the baseline model.

First Best. - If there is no idiosyncratic risk, $\sigma=0$, we get closed-form expressions for $r$ and $\hat{x}$

$$
\begin{gathered}
r=a-\delta \\
\hat{x}=(a-\delta-\rho) \psi+\delta
\end{gathered}
$$

Incomplete risk sharing and no money, $\beta=0$. - The non-monetary economy also allows for closed-form expressions, because $\lambda=\beta=0$ and $\sigma_{c}=\sigma$.

$$
\begin{gathered}
r=\underbrace{a-\delta}_{\text {first best }}-\gamma \sigma^{2} \\
\hat{x}=\underbrace{(a-\delta-\rho) \psi+\delta}_{\text {first best }}+\psi[\underbrace{(1+1 / \psi)(\gamma / 2) \sigma^{2}}_{\text {precautionary }}-\underbrace{\gamma \sigma^{2}}_{\text {risk pr. }}]
\end{gathered}
$$

After a risk shock increases idiosyncratic risk $\sigma$, the real interest rate falls to accommodate the higher risk premium $\alpha=\gamma \sigma_{c} \sigma=\gamma \sigma^{2}$. But investment may go up or down, depending on the intertemporal elasticity $\psi$. If $\psi>1$, investment falls when idiosyncratic risk $\sigma$ goes up; if $\psi<1$, investment raises. This can be understood in terms of the risk premium and precautionary motive. If $\psi>1$, the precautionary motive is smaller than the risk premium, and the difference increases with $\sigma((1+1 / \psi) / 2<1)$. Intuitively, capital is less attractive because it is more risky, and since agents are very intertemporally elastic, they substitute towards consuming instead (accepting a big change in the growth rate of their consumption). But if $\psi<1$, the precautionary motive dominates. Agents really want to smooth out their utility, and since they face more risk, they make it up by accumulating more capital. If $\psi=1$, as in the baseline, the two effects cancel out and investment does not change when $\sigma$ goes up.

The important variable is the intertemporal elasticity. Risk aversion, $\gamma$, just makes the idiosyncratic risk matter more. In fact, both enter jointly $\gamma \sigma^{2}$ in the equations. The role of intertemporal elasticity is well understood, and is the reason that the literature on time varying risk typically assumes high intertemporal elasticity, $\psi>1$. Empirically, evidence about $\psi$ is mixed, but $\psi=1$ is considered a quantitatively reasonable benchmark.

Incomplete risk sharing and money, $\beta>0$. - Now let's see what happens when we add money. First, take the liquidity share $\lambda>0$ as given. Idiosyncratic risk 
sharing improves, $\sigma_{c}=(1-\lambda) \sigma$, so we get

$$
\begin{gathered}
r=\underbrace{a-\delta}_{\text {first best }}-\gamma(1-\lambda) \sigma^{2} \\
\hat{x}=\underbrace{(a-\delta-\rho) \psi+\delta}_{\text {first best }}+\psi[\underbrace{(1+1 / \psi)(\gamma / 2) \sigma^{2}(1-\lambda)^{2}}_{\text {precautionary }}-\underbrace{\gamma \sigma^{2}(1-\lambda)}_{\text {risk pr. }}]
\end{gathered}
$$

Money weakens the risk premium, so the real interest rate is higher than without money. Money also weakens the precautionary motive more than the risk premium, just as in the baseline model. But since investment can go up or down with risk, depending on $\psi$, it is useful to decompose the effect of higher risk into the effect without money, and what money adds relative to the non-monetary economy:

$$
r=\underbrace{a-\delta-\gamma \sigma^{2}}_{\text {non-monetary }}+\underbrace{\lambda \gamma \sigma^{2}}_{\Delta r}
$$

$$
\hat{x}=\underbrace{(a-\delta-\rho) \psi+\delta+\psi\left[(1+1 / \psi)(\gamma / 2) \sigma^{2}-\gamma \sigma^{2}\right]}_{\text {non-monetary }}+\underbrace{\gamma \sigma^{2} \psi\left(\left(\lambda^{2}-2 \lambda\right)(1+1 / \psi) / 2+\lambda\right)}_{\Delta \hat{x}}
$$

The second terms are the effect of money on the real interest rate, $\Delta r$, and investment, $\Delta \hat{x}$, relative to the economy without money.

In general it is possible for investment in the monetary economy to be higher than in the non-monetary one. For very large $\psi, \Delta \hat{x} \approx \gamma \sigma^{2} \psi \lambda^{2} / 2>0$. There are two forces at work. Remember that if $\psi>1$, the risk premium dominates, so high risk $\sigma$ can have a very large negative effect on investment $\hat{x}$. Money improves risk sharing and weakens the risk premium $\alpha=\gamma \sigma^{2}(1-\lambda)$, so it dampens the fall in investment from this channel. It also weakens the precautionary motive relative to the risk premium, which reduces investment just like in the baseline model. The two forces work in opposite directions. In the baseline setting the direct effect of high risk in the absence of money is zero, so money must reduce investment.

For $\psi \leq 1$ we can obtain a clean characterization, such that the main properties of the baseline model go through. ${ }^{2}$ Money keeps the real interest rate from falling during downturns with high risk, and reduces investment. Risk aversion $\gamma$ and idiosyncratic risk $\sigma$ enter together as $\gamma \sigma^{2}$, so all our results apply as well to increases in risk aversion.

Proposition 1. For $\psi \leq 1$, the monetary economy has higher interest rate and lower investment than the non-monetary one; i.e. $\Delta r>0$ and $\Delta \hat{x}<0$. Higher $\gamma \sigma^{2}$ leads to higher risk premium $\alpha=\gamma \sigma^{2}(1-\lambda)$, higher liquidity share $\lambda$, and

\footnotetext{
${ }^{2} \psi \leq 1$ is sufficient, but not necessary.
} 
larger $\Delta r$ and $|\Delta \hat{x}|$.

The case $\psi \leq 1$ covers two very salient classes of preferences. First, CRRA preferences with risk aversion $\gamma=1 / \psi \geq 1$. This is the most common specification in macroeconomic models. In the context of models with time varying risk, it has the unappealing feature that, without money, higher risk leads to more investment.

Second, the cleanest and quantitatively salient specification has $\psi=1$ and $\gamma>1$. The $\gamma>1$ can be interpreted either as high risk aversion, or as ambiguity aversion as in Barillas, Hansen and Sargent (2009). This specification has the advantage that the equations boils down to those of the baseline model, with the only modification of replacing $\sigma^{2}$ by $\gamma \sigma^{2}$,

$$
\begin{gathered}
r=\underbrace{a-\delta-\gamma \sigma^{2}}_{\text {non-monetary }}+\underbrace{\lambda \gamma \sigma^{2}}_{\Delta r} \\
\hat{x}=\underbrace{(a-\rho)}_{\text {non-monetary }}-\underbrace{\rho \frac{\lambda-\beta}{1-\lambda}}_{\Delta \hat{x}} \\
\lambda=\frac{\rho \beta}{\rho-\gamma \sigma^{2}(1-\lambda)^{2}}
\end{gathered}
$$

PROOF OF PROPOSITION 1:

Write the equation for $\lambda$, replacing $r$ and $\hat{x}$ from (A.8) and (A.9) to obtain

$$
\lambda=\frac{\rho \beta}{\rho+(1 / \psi-1)(a-\delta-\rho) \psi-\alpha[(1-\psi)+\psi(1+1 / \psi)(1-\lambda) / 2]}
$$

where $\alpha=\gamma \sigma^{2}(1-\lambda)>0$ is the risk premium. The denominator is strictly decreasing in $\alpha$ (here we use $\psi \leq 1$ ) and strictly increasing in $\lambda$ (for $\alpha>0$ which must be the case for $\sigma>0)$. So if there is a solution $\lambda(\alpha)$ to this equation, it is increasing in $\alpha$. From $\alpha /(1-\lambda)=\gamma \sigma^{2}$, since the left hand side is increasing in $\alpha$, it follows that $\alpha$ is increasing in $\gamma \sigma^{2}$, and therefore so is $\lambda$.

Now $\Delta r=\lambda \gamma \sigma^{2}>0$ and increasing in $\gamma \sigma^{2}$ is straightforward. For $\Delta \hat{x}$ we write it after some algebra

$$
\Delta \hat{x}=\gamma \sigma^{2} \lambda\left(\lambda \frac{1+\psi}{2}-1\right)=\alpha \lambda \frac{\lambda \frac{1+\psi}{2}-1}{1-\lambda}<0
$$

If $\gamma \sigma^{2}$ increases, so does $\alpha$ and $\lambda$. The derivative of the last factor with respect to $\lambda$ is

$$
\partial_{\lambda}\left\{\frac{\lambda \frac{1+\psi}{2}-1}{1-\lambda}\right\}=\frac{\frac{1+\psi}{2}(1-\lambda)+\lambda \frac{1+\psi}{2}-1}{(1-\lambda)^{2}}=\frac{\frac{1+\psi}{2}-1}{(1-\lambda)^{2}} \leq 0
$$


(using $\psi \leq 1$ again). So $\Delta \hat{x}$ is negative and becomes even more negative.

\section{Contractual setting}

In this Appendix I develop the contractual environment that yields the incomplete idiosyncratic risk sharing problem in the baseline model as the optimal contract. I also allow aggregate risk with complete risk sharing, which is the setting in the dynamic model in Section III. The setting in the baseline model is a special case with no aggregate risk.

The setting is essentially a special case of the environment in Di Tella and Sannikov (2016) with perfect misreporting ( $\phi=1$ in the terms of that paper), generalized to allow for aggregate shocks. I discuss the similarities and differences below.

\section{A. Setting}

The setting is as in the dynamic model in Section III. The "capital quality" shock for an agent is

$$
\Delta_{i, t}^{k}=\sigma_{t} k_{i, t} d W_{i, t}+\tilde{\sigma}^{T F P} d Z_{t}^{T F P}
$$

where $Z^{T F P}$ is an aggregate TFP shock. Aggregate TFP risk $\tilde{\sigma}^{T F P}$ is constant, but idiosyncratic risk $\sigma_{t}$ follows an autoregressive process

$$
d \sigma_{t}=\mu_{\sigma}\left(\sigma_{t}\right) d t+\tilde{\sigma}_{\sigma}\left(\sigma_{t}\right) d Z_{t}^{R S}
$$

where $Z^{R S}$ is the aggregate risk shock. $Z^{T F P}$ and $Z^{R S}$ are independent Brownian motions.

There is a complete financial market with real interest rate $r$, nominal interest rate $i$, capital's excess return $\alpha$, and price of aggregate shocks $\theta^{T F P}$ and $\theta^{R S}$, all adapted to the history of aggregate shocks $Z^{T F P}$ and $Z^{R S}$. Let $Q$ be the equivalent martingale measure associated with $r, \theta^{T F P}$ and $\theta^{R S}$, and $\tilde{Q}$ the equivalent martingale measure associated with $r, \theta^{T F P}, \theta^{R S}$, and $\alpha .^{3}$

The agent receives consumption $c$ and money holdings $m$ from the principal, and manages capital $k$, all contingent on the history of aggregate shocks $Z^{T F P}$ and $Z^{R S}$ and the agent's report of his idiosyncratic shock $Y^{s}$. The idiosyncratic shock is not observable by the principal, so the agent can misreport at rate $s$, such that his reports are $Y_{t}^{s}=W_{t}-\int_{0}^{t} \frac{s_{u}}{\sigma_{u}} d u$. Furthermore, the agent has access to hidden trade that allows him to choose his consumption $\tilde{c}$, money $\tilde{m}$, capital holdings $\tilde{k}$, and to trade aggregate risk $\tilde{\sigma}_{n}^{T F P}$ and $\tilde{\sigma}_{n}^{R S} \cdot{ }^{4}$ His hidden savings $n$

\footnotetext{
${ }^{3}$ That is, $Q$ is defined by the SPD $d \xi_{t} / \xi_{t}=-r_{t}-\theta_{t}^{T F P} d Z_{t}^{T F P}-\theta_{t}^{R S} d Z_{t}^{R S}$ and $\tilde{Q}$ by $d \tilde{\xi}_{t} / \tilde{\xi}_{t}=$ $-r_{t}-\theta_{t}^{T F P} d Z_{t}^{T F P}-\theta_{t}^{R S} d Z_{t}^{R S}-\frac{\alpha_{t}}{\sigma_{t}} d W_{t}$.

${ }^{4}$ To keep things simple, allow $\tilde{k}<0$, but we can also restrict it to $\tilde{k} \geq 0$, as in Di Tella and Sannikov (2016). This doesn't change the optimal contract.
} 
start at $n_{0}=0$ and satisfy the dynamic budget constraint

$$
\begin{aligned}
d n_{t} & =\left(n_{t} r_{t}+c_{t}-\tilde{c}_{t}+\left(m_{t}-\tilde{m}_{t}\right) i_{t}+\left(\tilde{k}_{t}-k_{t}\right) \alpha_{t}+\theta_{t}^{T F P} \sigma_{n t}^{T F P}+\theta_{t}^{R S} \tilde{\sigma}_{n t}^{R S}+k_{t} s_{t}\right) d t \\
& +\left(\tilde{k}_{t}-k_{t}\right) \sigma_{t} d W_{t}+\tilde{\sigma}_{n t}^{T F P} d Z_{t}^{T F P}+\tilde{\sigma}_{n t}^{R S} d Z^{R S}
\end{aligned}
$$

with solvency constraint $n_{t} \geq \underline{n}_{t}$ where $\underline{n}_{t}$ is the natural debt limit

$$
\underline{n}_{t}=-\max _{s \in \mathbb{S}} \mathbb{E}_{t}^{\tilde{Q}}\left[\int_{t}^{\infty} e^{\int_{t}^{u} r_{\tau} d \tau}\left(c_{u}\left(Y^{s}\right)+m_{u}\left(Y^{s}\right) i_{u}+s_{u} k_{u}\left(Y^{s}\right)\right) d u\right]
$$

where $\mathbb{S}=\left\{s: \mathbb{E}^{\tilde{Q}}\left[\int_{0}^{\infty} e^{\int_{t}^{u} r_{\tau} d \tau}\left|c_{u}\left(Y^{s}\right)+m_{u}\left(Y^{s}\right) i_{u}+s_{u} k_{u}\left(Y^{s}\right)\right| d u\right]<\infty\right\}$ is the set of feasible stealing plans for a given contract. The natural debt limit $\underline{n}_{t}$ is the maximum amount that the agent can pay back for sure at time $t$. The lender is not taking any risk as long as he enforces the natural debt limit.

Lemma 1. Assume $\left|\underline{n}_{0}\right|<\infty$. If $n_{t} \geq \underline{n}_{t}$ always, then $\liminf \operatorname{in}_{t \rightarrow \infty} e^{-\int_{0}^{t} r_{u} d u} n_{t} \geq 0$ a.s.

A contract $\mathcal{C}=(c, m, k)$ is admissible if $\mathbb{E}^{Q}\left[\int_{0}^{\infty} e^{-\int_{0}^{t} r_{u} d u}\left|c_{t}+m_{t} i_{t}+k_{t} \alpha_{t}\right| d t\right]<$ $\infty$. It is always optimal to implement no misreporting or hidden trade. ${ }^{5}$ An admissible contract is incentive compatible if the agent chooses to report truthfully and not engage in hidden trade,

$$
(c, m, k, 0,0,0) \in \arg \max _{\mathcal{P}} U(c, m) \quad s t:
$$

where $\mathcal{P}=\left(\tilde{c}, \tilde{m}, \tilde{k}, \tilde{\sigma}_{n}^{T F P}, \tilde{\sigma}_{n}^{R S}, s\right)$. An incentive-compatible contract is optimal if it minimizes the cost of delivering utility to the agent

$$
\begin{gathered}
J_{0}\left(u_{0}\right)=\min _{(c, m, k) \in I C} \mathbb{E}^{Q}\left[\int_{0}^{\infty} e^{-\int_{0}^{t} r_{u} d u}\left(c_{t}+m_{t} i_{t}-k_{t} \alpha_{t}\right) d t\right] \\
\text { st }: \quad U(c, m) \geq u_{0}
\end{gathered}
$$

We pin down the agent's initial utility $u_{0}$ with a free-entry condition for principals. If the agent has initial wealth $w_{0}$, he gives it to the principal in exchange for the full-commitment contract, and the principal breaks even, $J_{0}\left(u_{0}\right)=w_{0}$.

\section{B. Incentive compatibility and optimal contract}

Given contract $\mathcal{C}=(c, m, k)$, the agent's problem is to choose a misreporting and hidden trade strategy $\mathcal{P}=\left(\tilde{c}, \tilde{m}, \tilde{k}, \tilde{\sigma}_{n}^{T F P}, \tilde{\sigma}_{n}^{R S}, s\right)$ to maximize his utility subject to his dynamic budget constraint. With the natural debt limit, the dynamic

${ }^{5}$ See Di Tella and Sannikov (2016). 
budget constraint is equivalent to the following intertemporal budget constraint (A.14)

$$
\mathbb{E}^{\tilde{Q}}\left[\int_{0}^{\infty} e^{-\int_{0}^{t} r_{u} d u}\left(\tilde{c}_{t}+\tilde{m}_{t} i_{t}\right) d t\right] \leq \max _{s \in \mathbb{S}} \mathbb{E}^{\tilde{Q}}\left[\int_{0}^{\infty} e^{-\int_{0}^{t} r_{u} d u}\left(c_{t}\left(Y^{s}\right)+m_{t}\left(Y^{s}\right) i_{t}+k_{t}\left(Y^{s}\right) s_{t}\right) d t\right]
$$

The right hand side is the present value of the agent's income from the principal, including what he "steals" from him, and is equal to (minus) the natural debt limit $-\underline{n}_{0}$. Of course, if the right hand side is infinity the agent can achieve infinite utility. This corresponds to the case where the natural debt limit $\underline{n}_{0}=-\infty$ so the agent can get infinite utility under the dynamic constraint as well.

Lemma 2. Assume $\left|\underline{n}_{0}\right|<\infty$. If $\left(\tilde{c}, \tilde{m}, \tilde{k}, \tilde{\sigma}_{n}^{T F P}, \tilde{\sigma}_{n}^{R S}, s\right)$ and $n$ satisfy the dynamic budget constraint (A.12) with $n_{t} \geq \underline{n}_{t}$ always, then $(\tilde{c}, \tilde{m})$ satisfy the intertemporal budget constraint (A.14).

If $(\tilde{c}, \tilde{m})$ satisfy the intertemporal budget constraint (A.14), then there are processes $\left(\tilde{k}, \tilde{\sigma}_{n}^{T F P}, \tilde{\sigma}_{n}^{R S}, s\right)$ and $n$ that satisfy the dynamic budget constraint (A.12) with $n_{t} \geq \underline{n}_{t}$ always.

We can split the agent's problem into two parts. First, pick a misreporting strategy that maximizes the value of the right hand side. Second, choose $\tilde{c}$ and $\tilde{m}$ to maximize utility subject to the intertemporal budget constraint (A.14).

If $s^{*}=0$ is optimal, then

$$
\int_{0}^{t} e^{-\int_{0}^{u} r_{\tau} d \tau}\left(c_{u}\left(Y^{s}\right)+m_{u}\left(Y^{s}\right) i_{u}+k_{u}\left(Y^{s}\right) s_{u}\right) d u-e^{-\int_{0}^{t} r_{u} d u} \underline{n}_{t}\left(Y^{s}\right)
$$

must be a $\tilde{Q}$-martingale for $s=0$ and a supermartingale for any other $s$. So we can write

$$
\begin{aligned}
d\left(e^{-\int_{0}^{t} r_{u} d u} \underline{n}_{t}\left(Y^{s}\right)\right) & =e^{-\int_{0}^{t} r_{\tau} d \tau}\left\{\left(c_{t}\left(Y^{s}\right)+m_{t}\left(Y^{s}\right) i_{t}\right) d t+\sigma_{\underline{n} t}\left(Y^{s}\right)\left(d Y_{t}^{s}+\alpha_{t} d t\right)\right. \\
& \left.+\tilde{\sigma}_{\underline{n} t}^{T F P}\left(Y^{s}\right)\left(d Z_{t}^{T F P}+\theta_{t}^{T F P} d t\right)+\tilde{\sigma}_{\underline{n} t}^{R S}\left(Y^{s}\right)\left(d Z_{t}^{R S}+\theta_{t}^{R S} d t\right)\right\}
\end{aligned}
$$

If the agent misreports $s$, then

$$
\begin{array}{r}
\int_{0}^{t} e^{-\int_{0}^{u} r_{\tau} d \tau}\left\{\left(c_{u}\left(Y^{s}\right)+m_{u}\left(Y^{s}\right) i_{u}+k_{u}\left(Y^{s}\right) s_{u}\right) d u-\left(c_{u}\left(Y^{s}\right)+m_{u}\left(Y^{s}\right) i_{u}\right) d u\right. \\
\left.-\sigma_{\underline{n} u}\left(Y^{s}\right)\left(d Y_{u}^{s}+\alpha_{u} d u\right)-\tilde{\sigma}_{\underline{n} u}^{T F P}\left(Y^{s}\right)\left(d Z_{u}^{T F P}+\theta_{u}^{T F P} d u\right)-\tilde{\sigma}_{\underline{n} u}^{R S}\left(Y^{s}\right)\left(d Z_{u}^{R S}+\theta_{u}^{R S} d u\right)\right\}
\end{array}
$$

or simplifying,

$$
\begin{array}{r}
\int_{0}^{t} e^{-\int_{0}^{u} r_{\tau} d \tau}\left\{k_{u}\left(Y^{s}\right) s_{u} d u-\sigma_{\underline{n} u}\left(Y^{s}\right)\left(d Y_{u}^{s}+\alpha_{u} d u\right)-\tilde{\sigma}_{\underline{n} u}^{T F P}\left(Y^{s}\right)\left(d Z_{u}^{T F P}+\theta_{u}^{T F P} d u\right)\right. \\
\left.-\tilde{\sigma}_{\underline{n} u}^{R S}\left(Y^{s}\right)\left(d Z_{u}^{R S}+\theta_{u}^{R S} d u\right)\right\}
\end{array}
$$


must be a $\tilde{Q}$-supermartingale. Since $d Y_{t}^{s}=d W_{t}-\frac{s t}{\sigma_{t}} d t$, this requires

$$
k_{t}+\sigma_{\underline{n} t} \frac{1}{\sigma_{t}}=0 \Longrightarrow \sigma_{\underline{n} t}=-k_{t} \sigma_{t}
$$

In other words, for every dollar the agent misreports he must lose a dollar in present value of future income.

Second, taking the right hand side of the intertemporal budget constraint as given and choosing $\tilde{c}$ and $\tilde{m}$ is a standard consumption-portfolio problem. The FOC are

$$
\begin{array}{rlr}
\mu_{c t} & =r_{t}-\rho+\sigma_{c t}^{2}+\left(\tilde{\sigma}_{c t}^{T F P}\right)^{2}+\left(\tilde{\sigma}_{c t}^{R S}\right)^{2} & \text { Euler equation } \\
\alpha_{t} & =\sigma_{c t} \sigma_{t} & \text { demand for capital } \\
\tilde{\sigma}_{c t}^{T F P} & =\theta_{t}^{T F P} & \text { TFP shocks } \\
\tilde{\sigma}_{c t}^{R S} & =\theta_{t}^{R S} & \text { risk shocks } \\
m_{t} / c_{t} & =\beta /(1-\beta) i_{t}^{-1} & \text { money }
\end{array}
$$

In addition, optimality and zero hidden savings, $n_{t}=0$, imply that

$\mathbb{E}_{t}^{\tilde{Q}}\left[\int_{t}^{\infty} e^{-\int_{t}^{u} r_{\tau} d \tau}\left(\tilde{c}_{u}+\tilde{m}_{u} i_{u}\right) d u\right]=\mathbb{E}_{t}^{\tilde{Q}}\left[\int_{t}^{\infty} e^{-\int_{t}^{u} r_{\tau} d \tau}\left(c_{u}\left(Y^{0}\right)+m_{u}\left(Y^{0}\right) i_{u}\right) d t\right]=-\underline{n}_{t}$

and $c_{t}=(1-\beta) \rho \mathbb{E}_{t}^{\tilde{Q}}\left[\int_{t}^{\infty} e^{-\int_{t}^{u} r_{\tau} d \tau}\left(\tilde{c}_{u}+\tilde{m}_{u} i_{u}\right) d u\right]=(1-\beta) \rho\left(-\underline{n}_{t}\right)$. From this it follows that the geometric volatility of consumption is

$$
\sigma_{c t}=(1-\beta) \rho \frac{k_{t}}{c_{t}} \sigma_{t} \quad \text { "skin in the game" }
$$

It's worth noting that, given (A.15)-(A.19), the agent's continuation utility

$$
U_{t}=\mathbb{E}_{t}\left[\int_{t}^{\infty} e^{-\rho u}\left((1-\beta) \log c_{u}+\beta \log m_{u}\right) d u\right]
$$

will admit a representation $U_{t}=A_{t}+\frac{1}{\rho} \log c_{t}$. The skin in the game constraint (A.20) then implies $\sigma_{U t}=(1-\beta) c_{t}^{-1} k_{t} \sigma_{t}$. If the agent misreports a dollar and immediately consumes it he gets marginal utility $(1-\beta) c_{t}^{-1}$, so his continuation utility must go down by that amount to deter him.

Putting these conditions together we obtain the following result.

Lemma 3. An incentive-compatible contract $\mathcal{C}=(c, m, k)$ must satisfy conditions (A.15)-(A.20).

The incentive compatibility conditions (A.15)-(A.20) are necessary. In general, proving that they are sufficient for global incentive compatibility is a difficult 
problem, because the hidden trade allows the agent a large set of deviations. The strategy is to first characterize the optimal contract subject only to the necessary incentive compatibility constraints, and then prove that it is indeed incentive compatible. As it turns out, this will be straightforward in this setting because the optimal contract will coincide with letting the agent choose his own consumption, money, and capital (the optimal contract coincides with autarky).

We say that contract $\mathcal{C}=(c, m, k)$ solves the portfolio problem for $w_{0}>0$ if it maximizes $U(c, m)$ subject to the dynamic budget constraint

$$
\begin{aligned}
d w_{t} & =\left(r_{t} w_{t}-c_{t}-m_{t} i_{t}+k_{t} \alpha_{t}+\theta_{t}^{T F P} \tilde{\sigma}_{w t}^{T F P}+\theta_{t}^{R S} \tilde{\sigma}_{w t}^{R S}\right) d t+k_{t} \sigma d W_{t} \\
& +\tilde{\sigma}_{w t}^{T F P} d Z_{t}^{T F P}+\tilde{\sigma}_{w t}^{R S} d Z_{t}^{R S}
\end{aligned}
$$

with solvency constraint $w_{t} \geq 0$. This dynamic budget constraint is equivalent to

$$
\mathbb{E}^{\tilde{Q}}\left[\int_{0}^{\infty} e^{-\int_{0}^{t} r_{u} d u}\left(c_{t}+m_{t} i_{t}\right) d t\right] \leq w_{0}
$$

It is well known that (A.15)-(A.19) are the FOCs for this portfolio problem, so we get the following result.

Theorem 1. Let $(c, m, k)$ be an optimal contract for initial utility $u_{0}$, with cost $J\left(u_{0}\right)$. Then $(c, m, k)$ solves the portfolio problem for $w_{0}=J\left(u_{0}\right)$.

Conversely, let $(c, m, k)$ solve the portfolio problem for some $w_{0}>0$. If in addition $\lim _{t \rightarrow \infty} \mathbb{E}\left[e^{-r t} w_{t}\right]=0$, then $(c, m, k)$ is an optimal contract for initial utility $u_{0}$ with $J\left(u_{0}\right)=w_{0}$.

Remark. The condition $\lim _{t \rightarrow \infty} \mathbb{E}\left[e^{-r t} w_{t}\right]=0$ must be satisfied in the competitive equilibrium in the paper.

\section{Comparison to Di Tella and Sannikov (2016)}

This setting is essentially the same as in Di Tella and Sannikov (2016), with hidden investment and perfect misreporting ( $\phi=1$ in the context of that paper). The main result here is Theorem 1, which is analogous to Lemma 28 in that paper. This is therefore a special case of the environment in that paper.

But there are some differences. First, here I allow aggregate risk shocks that affect the investment environment. The setting in Di Tella and Sannikov (2016) is stationary. Second, in Di Tella and Sannikov (2016) the agent faces a no-debt solvency constraint $n_{t} \geq 0$ on his hidden savings $n$. Here I allow the agent to borrow up to the natural borrowing limit, using his income from the contract. As it turns out the optimal contract is the same. The no-debt borrowing constraint relaxes the IC constraints, but the principal does not use this freedom in the optimal contract. Intuitively, with $n_{t} \geq 0$ the principal could backload the agent's consumption if he wanted. But what he really wants to do is to front load it.

Finally, here I allow the agent to short capital in his hidden investment, $\tilde{k}_{t}<0$ and to overreport returns, $s_{t}<0$. This is done for simplicity. In Di Tella and 
Sannikov (2016) hidden investment and misreporting must be non-negative, $k_{t} \geq$ 0 and $s_{t} \geq 0$, and the optimal contract is the same (for the special case with $\phi=1)$.

\section{Proofs}

\section{PROOF OF LEMMA 1:}

From the definition of the natural debt limit (A.13), if we take absolute value on both sides we get the following inequality

$$
\left|\underline{n}_{t}\right| \leq S_{t}=\mathbb{E}_{t}^{\tilde{Q}}\left[\int_{t}^{\infty} e^{\int_{t}^{u} r_{\tau} d \tau}\left|c_{u}\left(Y^{s^{*}}\right)+m_{u}\left(Y^{s^{*}}\right) i_{u}+s_{u}^{*} k_{u}\left(Y^{s^{*}}\right)\right| d u\right]<\infty
$$

where $s^{*}$ is the misreporting process that achieves the maximum in (A.13). The martingale representation theorem yields

$d\left(e^{-\int_{0}^{t} r_{u} d u} S_{t}\right)=-e^{-\int_{0}^{t} r_{u} d u}\left|c_{t}\left(Y^{s^{*}}\right)+m_{t}\left(Y^{s^{*}}\right) i_{t}+s_{t}^{*} k_{t}\left(Y^{s^{*}}\right)\right| d t+\tilde{Q}$-local mart. terms

We also know that $\lim _{T \rightarrow \infty} \mathbb{E}^{\tilde{Q}}\left[e^{-\int_{0}^{T} r_{u} d u} S_{T}\right]=0$. To see this, write

$S_{0}=\mathbb{E}_{0}^{\tilde{Q}}\left[\int_{0}^{T} e^{\int_{0}^{u} r_{\tau} d \tau}\left|c_{u}\left(Y^{s^{*}}\right)+m_{u}\left(Y^{s^{*}}\right) i_{u}+s_{u}^{*} k_{u}\left(Y^{s^{*}}\right)\right| d u\right]+\mathbb{E}^{\tilde{Q}}\left[e^{-\int_{0}^{T} r_{u} d u} S_{T}\right]$

and take the limit $T \rightarrow \infty$, using the MCT on the first term. It follows that $\lim _{T \rightarrow \infty} e^{-\int_{0}^{T} r_{u} d u} S_{T}$ exists and is zero almost surely (see Problem 3.16 in Karatzas and Shreve (2012)). Since $\left|\underline{n}_{t}\right| \leq S_{t}$, the same is true for $\underline{n}_{t}$, and since $n_{t} \geq \underline{n}_{t}$, we obtain $\liminf \operatorname{in}_{T \rightarrow \infty} e^{-\int_{0}^{t} r_{u} d u} n_{t} \geq 0$ a.s.

\section{PROOF OF LEMMA 2:}

In the first direction, use the dynamic budget constraint to compute

$$
\begin{aligned}
\mathbb{E}^{\tilde{Q}}\left[e^{-\int_{0}^{t} r_{u} d u} n_{t}\right] & =\mathbb{E}^{\tilde{Q}}\left[\int_{0}^{t} e^{-\int_{0}^{u} r_{\tau} d \tau}\left(c_{u}\left(Y^{s}\right)+m_{u}\left(Y^{s}\right) i_{u}+k_{u}\left(Y^{s}\right) s_{u}\right) d u\right] \\
& -\mathbb{E}^{\tilde{Q}}\left[\int_{0}^{t} e^{-\int_{0}^{u} r_{\tau} d \tau}\left(\tilde{c}_{u}+\tilde{m}_{u} i_{u}\right) d t\right]
\end{aligned}
$$

Subtract $\mathbb{E}^{\tilde{Q}}\left[e^{-\int_{0}^{t} r_{u} d u} \underline{n}_{t}\right]<\infty$ from both sides. Because $\underline{n}_{0}$ is the maximum 
value that the agent can get, we obtain an inequality:

$$
\begin{aligned}
& \mathbb{E}^{\tilde{Q}}\left[e^{-\int_{0}^{t} r_{u} d u}\left(n_{t}-\underline{n}_{t}\right)\right] \leq \max _{s} \mathbb{E}^{\tilde{Q}}\left[\int_{0}^{\infty} e^{-\int_{0}^{u} r_{\tau} d \tau}\left(c_{u}\left(Y^{s}\right)+m_{u}\left(Y^{s}\right) i_{u}+k_{u}\left(Y^{s}\right) s_{u}\right) d u\right] \\
&-\mathbb{E}^{\tilde{Q}}\left[\int_{0}^{t} e^{-\int_{0}^{u} r_{\tau} d \tau}\left(\tilde{c}_{u}+\tilde{m}_{u} i_{u}\right) d u\right] \\
& \mathbb{E}^{\tilde{Q}}\left[\int_{0}^{t} e^{-\int_{0}^{u} r_{\tau} d \tau}\left(\tilde{c}_{u}+\tilde{m}_{u} i_{u}\right) d u\right] \leq-\underline{n}_{0}-\mathbb{E}^{\tilde{Q}}\left[e^{-\int_{0}^{t} r_{u} d u}\left(n_{t}-\underline{n}_{t}\right)\right]
\end{aligned}
$$

Take the limit $t \rightarrow \infty$ and use $n_{t} \geq \underline{n}_{t}$ to obtain the intertemporal budget constraint (A.14).

In the other direction, define

$$
n_{t}=\underline{n}_{t}+\mathbb{E}^{\tilde{Q}}\left[\int_{t}^{\infty} e^{-\int_{t}^{u} r_{\tau} d \tau}\left(\tilde{c}_{u}+\tilde{m}_{u} i_{u}\right) d u\right] \geq \underline{n}_{t}
$$

Define $L_{t}=\mathbb{E}_{t}^{\tilde{Q}}\left[\int_{t}^{\infty} e^{-\int_{t}^{u} r_{\tau} d \tau}\left(\tilde{c}_{u}+\tilde{m}_{u} i_{u}\right) d u\right]$, so that $\int_{0}^{t} e^{-\int_{0}^{u} r_{\tau} d \tau}\left(\tilde{c}_{u}+\tilde{m}_{u} i_{u}\right) d u+$ $e^{-\int_{0}^{t} r_{\tau} d \tau} L_{t}$ is a $\tilde{Q}$-martingale. Likewise, $-\int_{0}^{t} e^{-\int_{0}^{u} r_{\tau} d \tau}\left(c_{u}\left(Y^{s^{*}}\right)+m_{u}\left(Y^{s^{*}}\right) i_{u}+\right.$ $\left.k_{u}\left(Y^{s^{*}}\right) s_{u}^{*}\right) d t+e^{-\int_{0}^{t} r_{\tau} d \tau} \underline{n}_{t}$ is also $\tilde{Q}$-martingale, where $s^{*}$ is the misreporting process that achieves the maximum. So we can write

$$
\begin{aligned}
d n_{t} & =\left(n_{t} r_{t}+c_{t}\left(Y^{s^{*}}\right)+m_{t}\left(Y^{s^{*}}\right) i_{t}+k_{t}\left(Y^{s^{*}}\right) s_{t}^{*}-\left(\tilde{c}_{t}+\tilde{m}_{t} i_{t}\right)\right) d t \\
& +\left(\sigma_{\underline{n} t}+\sigma_{L t}\right)\left(\alpha_{t} d t+d W_{t}\right)+\left(\tilde{\sigma}_{\underline{n} t}^{T F P}+\tilde{\sigma}_{L t}^{T F P}\right)\left(\theta_{t}^{T F P} d t+d \tilde{Z}_{t}^{T F P}\right)+\left(\tilde{\sigma}_{\underline{n} t}^{R S}+\tilde{\sigma}_{L t}^{R S}\right)\left(\theta_{t}^{R S} d t+d \tilde{Z}_{t}^{R S}\right)
\end{aligned}
$$

Letting $\sigma_{\underline{n} t}+\sigma_{L t}=\tilde{k}_{t}-k_{t}, \tilde{\sigma}_{\underline{n} t}^{T F P}+\tilde{\sigma}_{L t}^{T F P}=\tilde{\sigma}_{n t}^{T F P}$, and $\tilde{\sigma}_{\underline{n} t}^{R S}+\tilde{\sigma}_{L t}^{R S}=\tilde{\sigma}_{n t}^{R S}$, we obtain the dynamic budget constraint (A.12).

\section{PROOF OF LEMMA 3:}

Immediate from the argument in Section III.B, noting that incentive compatibility requires $\left|\underline{n}_{0}\right|<\infty$.

\section{PROOF OF THEOREM 1}

In the first direction, if $(c, m, k)$ is an optimal contract, then it must satisfy the local IC constraints (A.15)-(A.19), which are the FOC for the consumptionportfolio problem. So $c$ and $m$ solve the optimal portfolio problem for some initial $w_{0}$, with an associated wealth process $w$ that satisfies the dynamic budget constraint (A.21) and $w_{t} \geq 0$. Now the IC constraint (A.20) pins down the corresponding $k$. We know that $c_{t}=(1-\beta) \rho w_{t}$ in the portfolio problem, so (A.20) and (A.16) imply

$$
\sigma_{c}=\frac{\alpha_{t}}{\sigma_{t}}=(1-\beta) \rho \frac{k_{t}}{c_{t}} \sigma_{t}
$$




$$
\Longrightarrow \frac{k_{t}}{w_{t}}=\frac{\alpha_{t}}{\sigma_{t}^{2}}
$$

which is the expression for capital in the portfolio problem. Finally, we need to show that $w_{0}=J_{0}$. Integrate the dynamic budget constraint (A.21) and take expectations under $Q$ to obtain

$$
w_{0}=\mathbb{E}^{Q}\left[\int_{0}^{T} e^{-r t}\left(c_{t}+m_{t} i-k_{t} \alpha\right) d t\right]+\mathbb{E}^{Q}\left[e^{-r T} w_{T}\right]
$$

If we take the limit $T \rightarrow \infty$, the first term will converge to $\mathbb{E}\left[\int_{0}^{\infty} e^{-r t}\left(c_{t}+m_{t} i-k_{t} \alpha\right) d t\right]=$ $J_{0}$ (apply dominated converge theorem and use feasibility). For the second term, because everything is proportional to $w$, we must have $J_{t}=A w_{t}$ for some $A>0$ (it will be $A=1$ ). The continuation cost of the contract $J_{t}=$

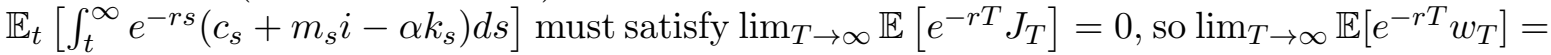
0 and therefore $w_{0}=J_{0}$. To see why $\lim _{T \rightarrow \infty} \mathbb{E}\left[e^{-r T} J_{T}\right]=0$, write $J_{0}=$ $\mathbb{E}\left[\int_{0}^{T} e^{-r t}\left(c_{t}+m_{t} i-k_{t} \alpha\right) d t\right]+\mathbb{E}\left[e^{-r T} J_{T}\right]$ and take $T \rightarrow \infty$ (using DCT and feasibility again).

In the other direction, suppose $(c, m, k)$ solve the portfolio problem with associated wealth process $w$ and utility utility $U(w)=\frac{1}{\rho}(\log w-\log \xi)$. Notice that this is the only contract that satisfies the local IC constraints and delivers utility $u_{0}=\frac{1}{\rho}\left(\log w_{0}-\log \xi\right)$. (A.15)-(A.19) are the FOC for the portfolio problem, and pin down $c$ and $m$ up to an initial constant (corresponding to $w_{0}$ ). We also know that $k_{t} / w_{t}=\frac{\alpha_{t}}{\sigma_{t}^{2}}$ and $c_{t}=(1-\beta) \rho w_{t}$, so from (A.16) we get the skin in the game IC constraint (A.20)

$$
\sigma_{c t}=\frac{\alpha_{t}}{\sigma_{t}}=\frac{k_{t}}{w_{t}} \sigma_{t}=(1-\beta) \rho \frac{k_{t}}{c_{t}} \sigma_{t}
$$

The contract $(c, m, k)$ is feasible because $\lim _{T \rightarrow \infty} \mathbb{E}\left[e^{-r T} w_{T}\right]=0$ (each term is proportional to $w$, so it grows slower than $r$ ). The contract is globally incentive compatible, because the agent is only getting risk-free debt from the principal, and doing what he wants.

It only remains to show that we can't achieve more utility. Integrate the dynamic budget constraint to obtain

$$
w_{0}=\mathbb{E}\left[\int_{0}^{T} e^{-r t}\left(c_{t}+m_{t} i-k_{t} \alpha\right) d t\right]+\mathbb{E}\left[e^{-r T} w_{T}\right]
$$

and take the limit $T \rightarrow \infty$ to obtain $J_{0}=w_{0}>0$. So giving the agent more utility (scaling up the contract) will cost more. 


\section{REFERENCES}

Barillas, Francisco, Lars Peter Hansen, and Thomas J Sargent. 2009. "Doubts or variability?" journal of economic theory, 144(6): 2388-2418.

Di Tella, Sebastian, and Yuliy Sannikov. 2016. "Optimal Asset Management Contracts with Hidden Savings." Stanford GSB.

Karatzas, Ioannis, and Steven Shreve. 2012. Brownian motion and stochastic calculus. Vol. 113, Springer Science \& Business Media.

Light, Bar. 2018. "Uniqueness of equilibrium in a Bewley-Aiyagari model." Economic Theory. 